\title{
Mesure et évaluation de la qualité des pratiques de développement des compétences informationnelles au sein du réseau de l'Université du Québec
}

\author{
GUY BÉLANGER \\ Université du Québec à Rimouski \\ Guy_Belanger@UQAR.ca \\ MARIE-MICHÈLE LEMIEUX \\ Université du Québec \\ marie-michele.lemieux@uquebec.ca \\ DENIS BOISVERT \\ Université du Québec à Rimouski \\ Denis_Boisvert@uqar.ca
}

\section{Résumé}

Une multitude de pratiques diversifiées ont été mises en œuvre afin de favoriser le développement des compétences informationnelles (CI) chez les étudiants du réseau de l'Université du Québec. Ces pratiques n'ont jamais fait l'objet d'évaluation de leur pertinence, ni des effets engendrés. Afin de contribuer à solutionner cette problématique, une étude méthodologique en trois phases a été conduite au sein de ce réseau. La première phase a permis de valider 339 critères de qualité des pratiques de développement des compétences informationnelles (PDCI) auprès d'experts. La deuxième phase consistait à expérimenter un processus de mesure de la qualité des formations documentaires et de la collaboration interprofessionnelle. Dans la troisième phase, des cercles de qualité constitués dans six universités ont procédé à l'évaluation de leurs résultats. La mise en commun de ces évaluations a permis d'identifier les forces et les points faibles significatifs en matière de PDCI. Les forces identifiées sont la qualité de la prestation et le niveau d'expertise démontrés par les bibliothécaires ainsi que leur relation avec les professeurs. Les points faibles se réfèrent à la faible collaboration interprofessionnelle et aux stratégies pédagogiques employées dans le cadre des formations documentaires. Une observation pour le moins paradoxale se dégage des résultats. Alors que la relation entre les bibliothécaires et les professeurs constitue la troisième 
force en importance, la collaboration interprofessionnelle a été identifiée comme le principal point faible. Ce constat a conduit les auteurs à définir un continuum de pratiques collaboratives permettant de préciser les différents niveaux de travail collaboratif.

\section{Summary}

A multitude of diversified practices have been established to facilitate the development of information literacy (IL) for students of the Université du Québec system. These practices have never been evaluated for relevance or effects. To contribute to the solution of this problem, a three-phase methodological study was carried out within this system. The first phase helped validate 339 criteria of quality of the practices of development of the information literacy (PDIL) with experts. The second consisted in experimenting a process to measure the quality of documentary training and the interprofessional collaboration. In the third phase, quality circles proceeded to the evaluation of the results obtained in six universities. The common grouping in these evaluations helped to identify the significant strengths and weaknesses in IL development practices. The strengths identified are the quality of the delivery by the librarians and their level of expertise as well as the relationship with the professors. The weak points refer to the low interprofessional collaboration and the pedagogic strategies used in the document training. An observation at least paradoxical emerges from the results. While the relationship between librarians and professors constitute the third most important force, interprofessional collaboration has been identified as the main weakness. This led the authors to define a continuum of collaborative practices to describe the different levels of collaborative work.

\section{Mots-clés}

Qualité, évaluation, collaboration, compétences informationnelles, interdisciplinarité

\section{Key words}

Quality, evaluation, collaboration, information skills, interdisciplinarity

\section{Introduction}

Dans un contexte d'enseignement et de recherche, où nous sommes passés en quelques années d'un problème d'accès à l'information à un problème d'excès (Karsenti et Dumouchel, 2011), il s'avère essentiel de former les étudiants à devenir info-compétents, c'est-à-dire de les rendre aptes à poser un regard critique sur la masse d'informations qui s'offre à eux pour qu'ils 
puissent l'exploiter judicieusement dans le cadre de leur cheminement universitaire. Afin d'amener progressivement l'étudiant à développer des compétences informationnelles (CI) reposant sur des normes reconnues, telles que proposées par l'Association of College \& Research Libraries (ACRL), l'intégration de la formation documentaire dans le curriculum a été reconnue comme étant le moyen «le plus prometteur et le plus efficace » (Université du Québec, 2004, p. 10). En l'espace d'une douzaine d'années (2003-2015), de multiples ressources et outils ont été développés et exploités dans le cadre de pratiques dénuées de tout processus d'évaluation de leurs qualités intrinsèques. Aussi le présent projet visait-il à évaluer la qualité des formations documentaires ainsi que les pratiques collaboratives interprofessionnelles liées au développement des CI chez les étudiants. Cette étude a permis de dresser un portrait des forces et des points faibles relatifs aux PDCI au sein du réseau de l’Université du Québec.

Dans l'ordre, cet article exposera la problématique qu'engendre l'absence d'évaluation de la qualité des PDCI dans un contexte où chacun des acteurs et services impliqués intervient isolément. Le cadre conceptuel permettra de préciser la posture épistémologique adoptée par les auteurs ainsi que les questions de recherche posées. La section consacrée à la méthodologie décrira les trois phases de l'étude. Les résultats obtenus seront présentés en précisant les forces et les points faibles identifiés au sein du réseau de l'Université du Québec en matière de PDCI. Finalement, la discussion permettra d'une part, d'établir des liens significatifs entre les forces et les points faibles identifiés, particulièrement en matière de collaboration interprofessionnelle et de stratégies pédagogiques, et d'autre part, de proposer des recommandations dans le but d'optimiser le développement de pratiques collaboratives entre les acteurs impliqués dans le développement des CI chez les étudiants universitaires.

\section{Problématique}

Une multitude de pratiques et de ressources technologiques en matière d'enseignement des CI ont été mises en œuvre dans le réseau de l'Université du Québec au cours des douze dernières années. Le problème à l'origine de cette étude provient du fait que peu d'études ont été consacrées à la mesure et à l'évaluation de la qualité des PDCI. Mentionnons qu'elles sont inexistantes au sein du réseau de l'Université du Québec, et ce, bien que les bénéfices associés au développement de ces compétences sur la réussite des étudiants soient reconnus (Julien et Boon, 2004 ; Julien et al., 2013 ; Samson, 2010). 
Plusieurs auteurs recommandent de mettre en place des pratiques de qualité en matière de développement des CI (ACRL, 2012 ; CAUL, 2004 ; Detlor et al., 2012 ; Detlor et al., 2011 ; Julien et Boon, 2004 ; Julien et al., 2013). Or, deux obstacles de taille se dressent devant un tel défi. D'une part, la représentation de ce que constitue des pratiques de qualité en matière de développement des CI ne fait pas consensus chez les auteurs, et d'autre part, il n'existe pas d'instrument de mesure de la qualité des PDCI. Un constat peut être établi : tous les acteurs veulent bien instaurer des pratiques de qualité en matière de développement des CI, mais personne n'est en mesure d'en évaluer la qualité à défaut d'instruments valides et surtout à défaut de considérer les effets obtenus et ce, dans la perspective d'un véritable travail collaboratif.

L'originalité de la présente étude repose sur l'adaptation et l'adoption d'un cadre intégrateur de la qualité des PDCI. Ce cadre intégrateur, issu du domaine de la qualité des services, permet d'une part, de prendre en compte des perspectives divergentes, et d'autre part, de préciser les relations entre les concepts déterminant la qualité des PDCI. Tel que proposé par Haddad et al. (1997), il a été jugé important de reconnaître tout d'abord la complexité du concept de qualité plutôt que de le réduire à une seule perspective.

\section{Cadre conceptuel}

La posture épistémologique adoptée par les auteurs relève du paradigme interprétativiste (Perret et Séville, 2007) dont le but principal consiste à donner un sens à des résultats et ce, en prenant en compte la nature subjective et contextuelle de la réalité. Il s'agissait ici, à partir des résultats de la mesure de la qualité, d'identifier les forces, les faiblesses (et leurs causes) en matière de PDCI au sein du réseau de l'Université du Québec. L'adoption de cette posture procurait deux avantages indéniables. D'une part, elle permettait d'exploiter un devis mixte, misant à la fois sur la collecte et l'analyse de données qualitatives et quantitatives. D'autre part, elle permettait de prendre en compte la nature subjective de la perspective des différents acteurs impliqués ainsi que le contexte particulier des différents établissements dans lesquels l'étude a été conduite. C'est en appui à ce paradigme que l'évaluation des résultats a d'abord été effectuée par des cercles de qualité au sein de chacun des établissements participants, et ce, en effectuant une évaluation de quatrième génération au sens de Guba et Lincoln (Fontan et Lachance, 2005 ; Guba et Lincoln, 1989). La Figure 1 présente le cadre de référence adopté par les auteurs. 


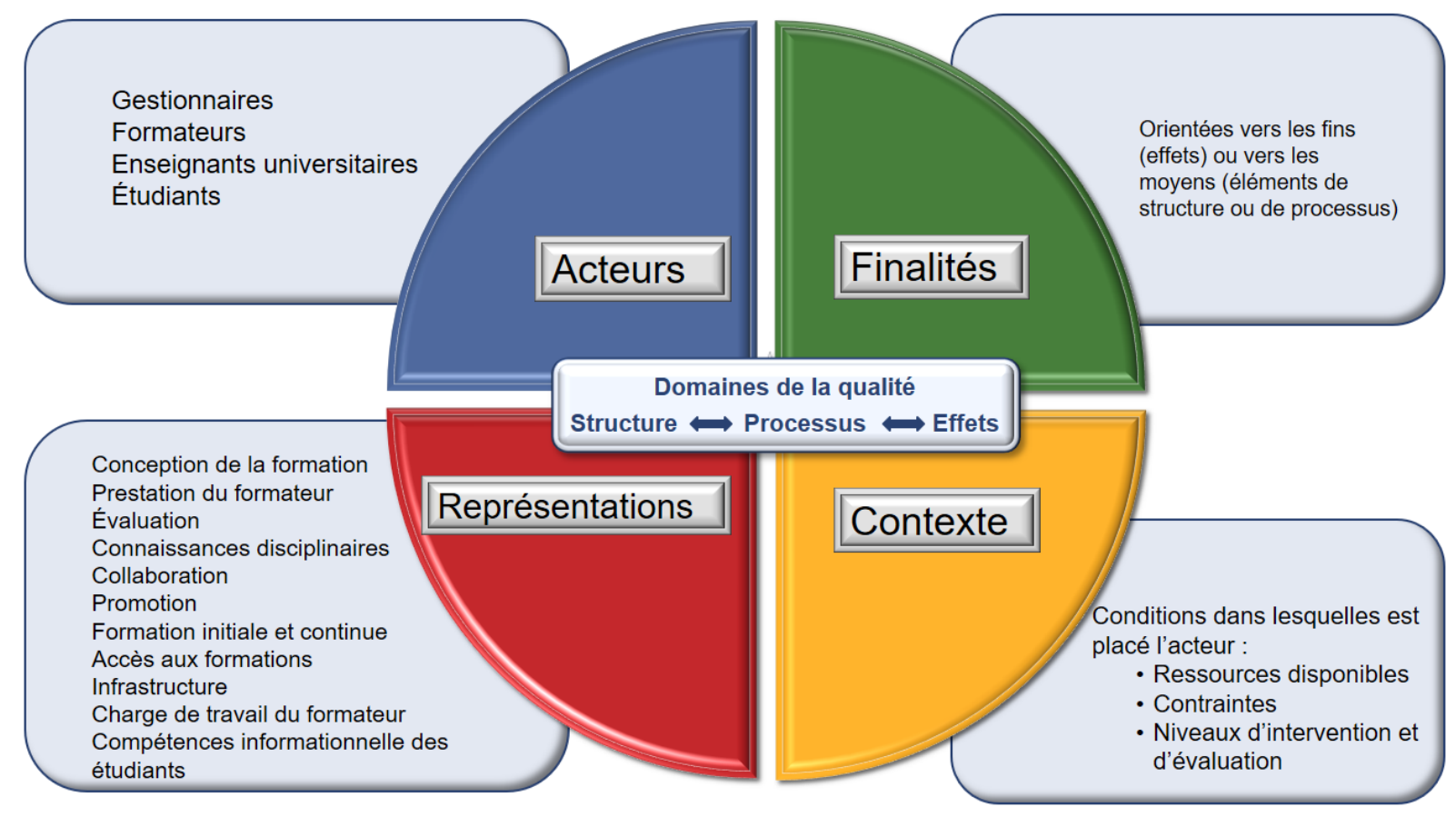

Figure 1 : modèle intégrateur de la qualité des pratiques de développement des compétences informationnelles (adapté de Bélanger, 2006)

Ce modèle conceptuel précise trois domaines d'évaluation de la qualité des PDCI : la structure (l'ensemble des ressources investies), les processus (stratégies pédagogiques - enseignement et apprentissage) et les effets obtenus en matière de développement des PDCI chez les étudiants universitaires. La force de cette conception repose sur le fait qu'elle permet de reconnaître et de prendre en compte la multiplicité des perspectives et représentations des différents acteurs impliqués. Ces représentations étant modulées en fonction du type d'acteur auquel on fait référence, des finalités poursuivies par ces acteurs et en fonction du contexte dans lequel sont placés ces acteurs. Cette conception permet d'exploiter la richesse de la multiplicité des perspectives dans la définition de ce que constituent des PDCI de qualité. Cette étude visait à répondre aux questions de recherche suivantes :

1. Quels sont, selon des bibliothécaires et des professeurs reconnus pour leur expertise en matière de pratiques de formation documentaire (PFD), les critères jugés suffisamment clairs, pertinents et importants pour opérationnaliser la mesure de la qualité des PFD au sein du réseau de l'Université du Québec?

2. Quelles sont les forces du réseau de l'Université du Québec en matière de PFD ?

3. Quelles sont les principales opportunités d'amélioration (points faibles) du réseau de l’Université du Québec en matière de PFD ?

\section{Méthodologie}


Cette étude s'est déroulée en trois phases. À partir d'une recension de plus de 1500 critères de qualité, l'utilisation de la technique DELPHI aura permis de valider 339 critères de qualité auprès de 18 bibliothécaires et 12 professeurs recrutés au sein du réseau de l'Université du Québec et reconnus par leurs pairs pour leur expertise en matière de développement des CI. Chacun des critères retenus devait démontrer un index de validité de contenu à l'échelle d'un item (Item Level - Content validity index [I-CVI]) d'au moins 0,8 (Lynn, 1986 ; Polit et Beck, 2006 ; Polit et al., 2007), tant pour leur clarté que pour leur pertinence et leur importance. Le processus de validation a nécessité deux consultations des experts. Au terme de l'exercice, une importante divergence de perspectives entre les experts bibliothécaires et professeurs a été identifiée au regard de l'évaluation des énoncés de critères qui leur ont été soumis. Cet aspect est davantage explicité dans le rapport de recherche produit par Bélanger et al. (2017).

La deuxième phase de l'étude a permis d'expérimenter un processus de mesure de la qualité des formations documentaires et de la collaboration interprofessionnelle. Pour ce faire, 146 critères de qualité ont pu être opérationnalisés dans le but de les expérimenter dans le cadre d'un processus de mesure. Parmi les critères opérationnalisés, 108 permettaient de mesurer la qualité des PFD à l'aide de cinq questionnaires administrés à 1760 étudiants en provenance de huit universités. Par ailleurs, 38 critères mesurant la qualité de la collaboration interprofessionnelle ont été soumis à 39 professeurs et 28 bibliothécaires.

La troisième phase de l'étude a permis d'expérimenter un processus d'évaluation de la qualité des PFD à partir des résultats générés lors de la mesure. Pour ce faire, des cercles de qualité ont été mis sur pied dans six universités. Chacun de ces cercles devait regrouper le directeur du Service de la bibliothèque, deux bibliothécaires, deux professeurs et deux étudiants. Ces cercles de qualité devaient, à partir des rapports qui leur ont été soumis, identifier et mettre en priorité les forces et les points faibles constatés au sein de leur établissement. Cet exercice a été conduit à l'aide d'un canevas inspiré du diagramme d'Ishikawa (2007). Ces cercles de qualité devaient également identifier et prioriser les principales causes à l'origine des points faibles observés et ce, dans le but de respecter le principe de Pareto qui stipule que $80 \%$ des problèmes sont engendrés par $20 \%$ des causes (ANAES, 2000). Lors de l'évaluation des résultats à l'échelle du réseau, c'est à l'aide de la technique de Blake et Mouton ${ }^{1}$, aussi appelée la technique du vote

1 La technique du vote pondéré consiste à attribuer une valeur à chacune des forces identifiées par un cercle de qualité selon un ordre décroissant de manière à respecter l'ordre de mise en priorité effectuée par chacun des cercles de qualité. La même valeur de départ a été attribuée à la première force identifiée par chacun des cercles de qualité afin d'assurer qu'elles aient toutes le même poids selon leur ordre d'importance. Dans le cas présent, 
pondéré (HAS, 2007), que le groupe de recherche a mis en perspective les résultats obtenus par chacune des universités avec ceux compilés à l'échelle du réseau, et ce, dans le but d'en dégager les tendances systémiques.

\section{Résultats}

Le tableau suivant présente les résultats obtenus pour chacune des catégories regroupant l'ensemble des critères mesurés.

Tableau 1: résultats obtenus dans l'ensemble du réseau de l'Université du Québec pour chacune des catégories de critères

\begin{tabular}{|c|c|c|}
\hline Facettes retenues pour regrouper les critères de qualité & $n$ critères & Résultats (\%) \\
\hline 1. Conception de la formation & & 74,9 \\
\hline 1.1 Objectifs d'apprentissage & 2 & 85,5 \\
\hline 1.2 Méthodes d'enseignement & 3 & 70,7 \\
\hline 1.3 Activités d'apprentissage & 14 & 72,8 \\
\hline 1.4 Structure des formations documentaires & 2 & 80,2 \\
\hline 2. Prestation & & 86,1 \\
\hline 2.1 Présentation des objectifs et du déroulement de la formation & 5 & 88,6 \\
\hline 2.2 Efficacité de la communication & & 85,5 \\
\hline 2.2.1 Stratégies favorisant la communication & 14 & 87,5 \\
\hline 2.2.2 Clarté de la présentation & 8 & 82,9 \\
\hline 2.2.3 Matériel de présentation & 9 & 88,0 \\
\hline 2.2.4 Interaction avec les étudiants & 8 & 81,6 \\
\hline 2.3 Gestion du temps & 5 & 88,6 \\
\hline 2.4 Disponibilité du formateur & 5 & 87,7 \\
\hline 2.5 Gestion des imprévus & 3 & 83,3 \\
\hline 3. Évaluation des apprentissages & 3 & 84,7 \\
\hline 4. Connaissances disciplinaires du formateur & 5 & 91,3 \\
\hline 5. Collaboration & & 58,5 \\
\hline 5.1 Collaboration avec l'enseignant universitaire & 32 & 59,5 \\
\hline 5.2 Collaboration entre formateurs & 3 & 52,1 \\
\hline 5.3 Collaboration (autres) & 3 & 21,8 \\
\hline 6. Formation initiale et continue & 4 & 67,5 \\
\hline 7. Infrastructure & 13 & 88,1 \\
\hline 8. Charge de travail du formateur & 5 & 46,5 \\
\hline Total : & 146 & 81,8 \\
\hline
\end{tabular}

C'est à partir de ces résultats globaux et des résultats spécifiques obtenus pour chacun des 146 critères mesurés que les six cercles de qualité devaient identifier les forces et les points faibles

puisque le plus grand nombre de forces identifiées par un cercle de qualité est 11, cette valeur a été retenue comme point de départ pour la première force identifiée par chacun des cercles de qualité. Par la suite, cette valeur a été décrémentée de 1 pour chacune des forces suivantes. La somme de ces valeurs attribuées aux forces associées à une catégorie représente la valeur pondérée de la catégorie. Plus cette valeur est importante, plus la force est jugée importante par l'ensemble des cercles de qualité. 
en matière de PDCI au sein de leur établissement. La mise en commun des rapports d'évaluation produits par ces cercles de qualité a permis de regrouper en cinq catégories l'ensemble des principales forces identifiées à l'échelle du réseau. Le Tableau 2 présente cette catégorisation.

Tableau 2 : catégories de forces identifiées au sein du réseau de l'Université du Québec en matière de PDCI, ordonnées selon leurs valeurs pondérées

\begin{tabular}{lc}
\hline $\begin{array}{c}\text { Catégories regroupant les forces } \\
\text { identifiées et priorisées par les } \\
\text { cercles de qualité }\end{array}$ & $\begin{array}{c}\text { Sommes des valeurs } \\
\text { pondérées des forces } \\
\text { regroupées }\end{array}$ \\
\hline Prestation du bibliothécaire & 171 \\
Compétences du bibliothécaire & 49 \\
Relations avec le professeur & 26 \\
Conception de la formation & 20 \\
Infrastructure & 13 \\
\hline
\end{tabular}

La catégorie de forces Prestation du bibliothécaire dans le cadre des formations documentaires se réfère aux stratégies de communication efficaces, à la présentation des objectifs et au déroulement de la formation, à la bonne relation avec les étudiants et à la disponibilité du bibliothécaire ainsi qu'à la bonne gestion du temps et à la clarté du matériel de présentation. La catégorie Compétences du bibliothécaire regroupe les forces précisant les connaissances disciplinaires du bibliothécaire ainsi que la maîtrise des technologies de l'information et des notions enseignées. La catégorie Relations avec les professeurs se réfère principalement à la qualité de la communication entre les professeurs et les bibliothécaires. La catégorie Conception de la formation regroupe les forces identifiant l'adéquation de la formation avec les objectifs d'apprentissage et les méthodes d'enseignement qui facilitent la compréhension des notions enseignées. Enfin, la catégorie Infrastructure concerne la qualité des locaux, des laboratoires et des équipements informatiques.

Le Tableau 3 présente une catégorisation des points faibles en matière de PDCI identifiés par les cercles de qualité des six universités participantes. On y retrouve également les causes qui ont été associées aux trois points faibles présentant les valeurs pondérées les plus fortes.

Tableau 3 : catégories de points faibles et leurs causes identifiées au sein du réseau de l'Université du Québec en matière de PDCI ordonnées selon leurs valeurs pondérées 


\begin{tabular}{|c|c|c|c|}
\hline $\begin{array}{c}\text { Catégories regroupant les } \\
\text { principaux points faibles } \\
\text { identifiés et priorisés par les } \\
\text { cercles de qualité }\end{array}$ & $\begin{array}{c}\text { Sommes des } \\
\text { valeurs } \\
\text { pondérées des } \\
\text { points faibles } \\
\text { regroupés }\end{array}$ & $\begin{array}{c}\text { Catégories regroupant les principales causes } \\
\text { identifiées et priorisées par les cercles de } \\
\text { qualité }\end{array}$ & $\begin{array}{c}\text { Sommes des } \\
\text { valeurs } \\
\text { pondérées des } \\
\text { causes } \\
\text { regroupées }\end{array}$ \\
\hline \multirow{5}{*}{ Collaboration } & \multirow{5}{*}{37} & Culture et pratiques organisationnelles & 45 \\
\hline & & Absence de planification & 30 \\
\hline & & Faible promotion des services & 23 \\
\hline & & Manque de temps & 21 \\
\hline & & Infrastructure & 10 \\
\hline \multirow{8}{*}{ Méthodes pédagogiques } & \multirow{8}{*}{33} & Manque de formation & 30 \\
\hline & & Faible collaboration interdisciplinaire & 26 \\
\hline & & Absence d'évaluation & 21 \\
\hline & & Infrastructure & 16 \\
\hline & & Temps alloué aux formations documentaires & 12 \\
\hline & & Absence d'activités d'apprentissage & 12 \\
\hline & & Manque de planification/coordination & 11 \\
\hline & & Aisance pédagogique & 3 \\
\hline \multirow{3}{*}{ Charge de travail du bibliothécaire } & \multirow{3}{*}{8} & Logistique & 9 \\
\hline & & Manque de ressources & 6 \\
\hline & & Manque d'organisation/coordination & 5 \\
\hline
\end{tabular}

Le manque de collaboration a été identifié comme principal point faible au sein du réseau. Il concerne plus spécifiquement le degré de collaboration entre les bibliothécaires et les professeurs, entre les bibliothécaires eux-mêmes et avec les autres professionnels impliqués dans les PDCI. La catégorie de points faibles «Collaboration » se réfère aussi au fait que les formations documentaires sont peu intégrées au cursus universitaire des étudiants. La catégorie de points faibles «Méthodes pédagogiques » fait référence aux stratégies pédagogiques adoptées par les bibliothécaires dans leurs enseignements ainsi qu'à la diversification des activités d'apprentissage offertes aux étudiants. La catégorie Charge de travail du bibliothécaire concerne le peu de temps pour planifier, offrir et effectuer des activités d'évaluation ainsi que le manque de disponibilité pour entreprendre des projets de collaboration.

\section{Discussion}

Une observation pour le moins paradoxale se dégage des résultats obtenus au sein de chacune des universités participantes. Alors que la relation entre les bibliothécaires et les professeurs constitue la troisième force en importance, la collaboration interprofessionnelle a été identifiée comme le principal point faible. De plus, les cercles de qualité ont identifié le manque de collaboration comme étant la deuxième cause en importance liée à la catégorie de points faibles Méthodes pédagogiques. Force est de reconnaître que l'absence d'un véritable travail collaboratif nous est rapidement apparu à l'origine de la plupart des problèmes identifiés dans le cadre de cette étude ; formations documentaires non intégrées au cursus, nombre élevé de formations documentaires diversifiées, absence de cadre commun de référence, peu de 
coordination des interventions multidisciplinaires, travail en silo. Pour cette raison, il devenait pertinent et essentiel de définir ce que constitue véritablement un travail dit collaboratif. Pour ce faire, l'intégration des modélisations de Little (1990, cité dans Beaumont, Lavoie et Couture, 2010), de Levan (2016) et de Piquet (2009) a permis de développer un continuum de pratiques de travail collaboratif permettant de définir les différents degrés d'évolution d'une véritable collaboration au sein d'une équipe. La figure 2 présente ce continuum.

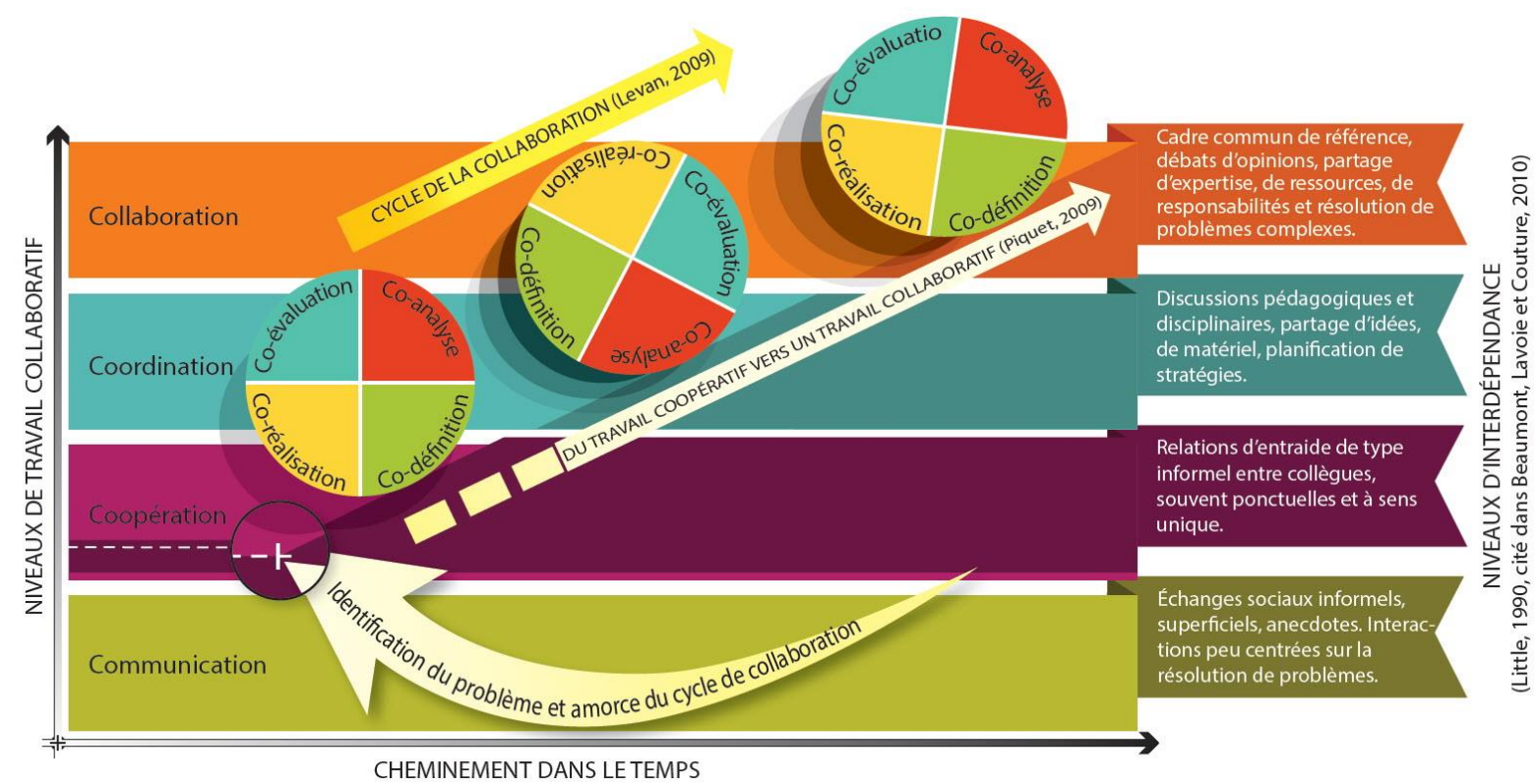

Figure 2 : le développement d'une approche collaborative interdisciplinaire

Cette modélisation permet de lever l'ambiguïté soulevée dans les résultats obtenus et de ce fait, de mieux comprendre comment les relations entre les bibliothécaires et les professeurs, se limitant principalement à des échanges sociaux informels, peuvent représenter une force, alors que la collaboration interprofessionnelle a été identifiée comme étant le principal point faible. Comme le précise Little (1990 ; voir Beaumont et al., 2010), une véritable collaboration repose sur l'adoption d'un cadre commun de référence, sur des débats d'opinions, de partage d'expertise, de ressources, de responsabilités. Il s'agit, en fait, de développer une relation d'interdépendance entre tous les acteurs impliqués dans la résolution de problèmes complexes tels que le développement des CI chez les étudiants universitaires. Cette modélisation permet également de réaliser que le développement d'une véritable pratique collaborative repose sur la mise en ouvre de ce que Levan (2016) appelle le cycle de la collaboration. Il s'agit d'un processus itératif dans lequel les acteurs s'impliquent activement dans des activités de coanalyse de la situation dans le but de développer un référentiel cognitif commun, de codéfinition des objectifs à poursuivre et de la stratégie à adopter, de co-réalisation des actions prévues pour atteindre les objectifs, et finalement, de co-évaluation des résultats obtenus. 
La catégorie de points faibles identifiée «Méthodes pédagogiques » constitue le deuxième problème en importance identifié par les cercles de qualité. Ce point faible se réfère principalement aux méthodes d'enseignement actuellement exploitées par les bibliothécaires, aux stratégies pédagogiques peu axées sur l'apprentissage des étudiants et à la densité du contenu « livré » pendant les formations documentaires. D'ailleurs, l'analyse effectuée par les différents cercles de qualité confirme un constat que nous avons émis lors de l'analyse des résultats du processus de validation des critères de qualité à l'effet que la perspective des bibliothécaires est davantage orientée vers un contenu à livrer lors des formations documentaires (perspective davantage axée sur les processus), plutôt que sur les effets recherchés en référence au développement des CI.

Les résultats de cette étude ont conduit les auteurs à formuler quatre grandes recommandations dans le but d'améliorer la qualité des PDCI. La première suggère d'initier un changement de paradigme en matière de PDCI tel que le précise le Tableau 4.

Tableau 4 : pratiques actuelles et préconisées en matière de développement des compétences informationnelles

\begin{tabular}{|c|c|c|}
\hline & Pratiques actuelles & Pratiques préconisées \\
\hline \multirow{2}{*}{$\begin{array}{l}\text { Degré } \\
\text { d'interdépendance } \\
\text { entre les différents } \\
\text { intervenants }\end{array}$} & Travail en vase clos (en silo) & $\begin{array}{l}\text { Travail collaboratif basé sur une relation } \\
\text { d'interdépendance }\end{array}$ \\
\hline & $\begin{array}{l}\text { Interventions ponctuelles multidisciplinaires (et } \\
\text { multiservices) }\end{array}$ & Approche interdisciplinaire coordonnée et collaborative \\
\hline Cadre de référence & $\begin{array}{l}\text { Les compétences informationnelles se réfèrent } \\
\text { principalement à la recherche documentaire }\end{array}$ & $\begin{array}{l}\text { Les compétences informationnelles se réfèrent aussi à } \\
\text { l'exploitation disciplinaire de l'information et à la gestion } \\
\text { des contenus (cadre référentiel de I'ACRL, 2016) incluant les } \\
\text { compétences informationnelles requises sur le marché du } \\
\text { travail }\end{array}$ \\
\hline \multirow{3}{*}{$\begin{array}{l}\text { Stratégies } \\
\text { pédagogiques }\end{array}$} & $\begin{array}{l}\text { Formations documentaires basées sur un contenu à } \\
\text { transmettre }\end{array}$ & $\begin{array}{l}\text { Formations documentaires adaptées aux besoins } \\
\text { d'apprentissage des étudiants }\end{array}$ \\
\hline & Pratiques axées sur l'acquisition de connaissances & Pratiques axées sur le développement de compétences \\
\hline & $\begin{array}{l}\text { Les bibliothécaires-formateurs et les enseignants } \\
\text { universitaires sont responsables de l'enseignement }\end{array}$ & $\begin{array}{l}\text { Les étudiants deviennent responsables de leur } \\
\text { apprentissage }\end{array}$ \\
\hline $\begin{array}{l}\text { Qualité des } \\
\text { pratiques }\end{array}$ & $\begin{array}{l}\text { Accent porté principalement sur la qualité des } \\
\text { processus en matière de pratiques de formation } \\
\text { documentaire }\end{array}$ & $\begin{array}{l}\text { Établissement de liens significatifs entre les éléments de } \\
\text { structure, de processus et d'effets obtenus en matière de } \\
\text { qualité des pratiques de développement des compétences } \\
\text { informationnelles }\end{array}$ \\
\hline
\end{tabular}

La deuxième recommandation concerne la création d'un espace de coordination et d'intégration des pratiques visant le développement des CI dans lequel une équipe multidisciplinaire, intégrant des représentants étudiants, aurait à concevoir et à mettre en œuvre un programme de développement des $\mathrm{CI}$ au sein de chaque établissement. Un tel programme permettrait de planifier et de coordonner une intervention collaborative interdisciplinaire en matière de PDCI. C'est également cette équipe qui aurait à mettre en application le cycle de la collaboration 
proposé par Levan (2016), et ce, dans le but d'analyser la situation, de définir les objectifs à poursuivre, de superviser la réalisation des activités et d'évaluer les résultats obtenus. La troisième recommandation propose de mettre en œuvre un programme d'amélioration continue de la qualité des PDCI dans le but d'en mesurer et d'en évaluer la qualité de manière périodique. Cet exercice peut se concrétiser par la conduite d'audits permettant de mesurer la qualité intrinsèque des éléments de structure et de processus mis en œuvre ainsi que les résultats effectivement obtenus chez les étudiants en matière de développement des CI. Finalement, la quatrième recommandation propose d'exploiter des outils pédagogiques collaboratifs optimisant le développement des CI. Ce type d'outil aiderait à définir un cadre commun de référence en matière de PDCI, à partager des ressources pédagogiques destinées tant à l'enseignement qu'à l'apprentissage des CI et idéalement, à fournir aux étudiants un outil guidant l'évolution du développement de leurs CI en fonction de leur programme d'étude.

\section{Conclusion}

La présente étude méthodologique visait à expérimenter des processus de mesure et d'évaluation de la qualité des PDCI. Pour ce faire, 339 critères de qualité ont été validés, des exercices de mesure de la qualité des formations documentaires et de la collaboration interprofessionnelle ont été expérimentés. Les résultats ont été interprétés par six cercles de qualité constitués dans six universités. L'évaluation des résultats obtenus démontre un important problème de collaboration entre les différents acteurs impliqués dans le développement des CI chez les étudiants. Afin de contrer ce problème, un virage s'impose. Il faut revoir nos façons de voir (le quoi), nos façons de faire (le comment) et surtout, de bien identifier nos finalités (le pourquoi). Pour y parvenir, nous proposons d'effectuer un changement de paradigme, de mettre en place un espace de collaboration interdisciplinaire et de mettre en œuvre un programme d'amélioration continue de la qualité des PDCI dans lequel des audits permettraient d'en mesurer la qualité et en particulier, les effets obtenus en matière de développement des CI chez les étudiants. Ce n'est qu'en instaurant une véritable pratique collaborative que l'on parviendra à établir un cadre commun de référence précisant les finalités poursuivies par une équipe multidisciplinaire en matière de développement des CI.

\section{Références bibliographiques}

ACRL. (2012). Characteristics of Programs of Information Literacy that Illustrate Best Practices: A Guideline. http://www.ala.org/acrl/standards/characteristics 
ANAES. (2000). Méthodes et outils des démarches qualité pour les établissements de santé. Paris : Agence nationale d'accréditation et d'évaluation en santé.

Beaumont, C., Lavoie, J. et Couture, C. (2010). Les pratiques collaboratives en milieu scolaire : cadre de référence pour soutenir la formation. Centre de recherche et d'intervention sur la réussite scolaire (CRIRES). Québec : Université Laval.

Bélanger, G. (2006). Validation nominale des critères mesurant la qualité des services de soutien à domicile selon la perspective des utilisateurs (Thèse de doctorat). Université de Montréal, Département d'Administration de la santé de la Faculté de médecine.

Bélanger, G., Boisvert, D., Lemieux, M.-M. et Séguin, C. (2017). Évaluation de la qualité des pratiques de développement des compétences informationnelles chez les étudiants du réseau de l'Université du Québec. Québec : Université du Québec. Rapport numérique en libre accès publié sur http://rapport-qualite-pdci.uquebec.ca/ et sur https://www.researchgate.net/publication/320501957 Rapport-FODAR-Cl-2017

CAUL. (2004). Best Practices Characteristics for Developing Information Literacy in Australian Universities : A Guideline. Australie.

Detlor, B., Booker, L., Serenko, A. et Julien, H. (2012). Student Perceptions of Information Literacy Instruction : The Importance of Active Learning. Education for Information, 29(2), 147-161.

Detlor, B., Julien, H., Willson, R., Serenko, A. et Lavallée, M. (2011). Learning outcomes of information literacy instruction at business schools. Journal of the American Society for Information Science and Technology, 62(3), 572-585.

Fontan, J.-M. et Lachance, É. (2005). Pour une évaluation de cinquième génération. Cahiers de l'ARUC-ÉS. Cahier $N^{\circ}$ C-18-2005. http://www.aruc-es.uqam.ca/Portals/0/cahiers/C-182005.pdf

Guba, E.G. et Lincoln, Y.S. (1989). Fourth generation evaluation. Newbury Park : Sage Publications.

Haddad, S., Roberge, D. et Pineault, R. (1997). Comprendre la qualité : en reconnaître la complexité. Ruptures, revue transdisciplinaire en santé, 4(1), 59-78.

HAS (Éd.). (2007). Guide méthodologique - Élaboration de critères de qualité pour l'évaluation et l'amélioration des pratiques professionnelles. France : Haute autorité de santé.

Ishikawa, K. (2007). La gestion de la qualité : Outils et applications pratiques. Paris : Dunod.

Julien, H. et Boon, S. (2004). Assessing instructional outcomes in Canadian academic libraries. Library \& Information Science Research, 26, 121-139.

Julien, H., Detlor, B. et Serenko, A. (2013). Information literacy in the business school context : A story of complexity and success. Dans M. Hepworth et G. Walton (Eds.), Developing people's information capabilities : Fostering information literacy in educational, workplace and community contexts (p. 167-177). Bingley : Émerald Group Publishing Lte.

Karsenti, T. et Dumouchel, G. (2011). Former aux compétences informationnelles au Québec : une mission partagée. Dans I. Fabre (dir.), Professeur-documentaliste : un tiers-métier (p. 87-109). Dijon : Educagri Éditions.

Levan, S.K. (2016). Ce que travail collaboratif veut dire... http://travailcollaboratif.typepad.com/methode main/2009/03/ce-que-travail-collaboratifveut-dire.html 
Lynn, M. R. (1986). Determination and quantification of content validity. Nursing Research, 35(6), 382-385.

Perret, V. et Séville, M. (2007). Fondements épistémologiques de la recherche. Dans R.-A. Thiétart (dir.), Méthodes de recherche en management (p. 13-33). Paris : Dunod.

Piquet, A. (2009). Guide pratique du travail collaboratif: Théories, méthodes et outils au service de la collaboration. http://a-

brest.net/IMG/pdf/Guide pratique du travail_collaboratif.pdf

Polit, D.F. et Beck, C.T. (2006). The content validity index : are you sure you know what's being reported? Critique and recommendations. Research in Nursing \& Health, 29(5), 489497.

Polit, D.F., Beck, C.T. et Owen, S.V. (2007). Is the CVI an acceptable indicator of content validity? Appraisal and recommendations. Research in Nursing \& Health, 30(4), 459-467.

Samson, S. (2010). Information Literacy Learning Outcomes and Student Success. The Journal of Academic Librarianship, 36(3), 202-210.

Université du Québec. (2004). La formation aux compétences informationnelles : une action fondamentale essentielle à la réussite de l'étudiant. Document d'orientation adopté par le Comité de pilotage le 14 mai 2004 (document de travail). Québec : Université du Québec. 